\title{
इㄴ. \\ Hemispheric asymmetries of memory: the HERA model revisited
}

\author{
Reza Habib ${ }^{1}$, Lars Nyberg ${ }^{2}$ and Endel Tulving ${ }^{1}$ \\ ${ }^{1}$ Rotman Research Institute, Baycrest Centre for Geriatric Care, 3560 Bathurst Street, Toronto, Ontario, Canada M6A 2E1. \\ ${ }^{2}$ Department of Psychology, Umeå University, Umeå S901-87, Sweden.
}

\begin{abstract}
The hemispheric encoding/retrieval asymmetry (HERA) model is a process-specific description of experimental data provided by a large set of functional neuroimaging studies. According to HERA, left prefrontal cortex (PFC) is more involved than right PFC in episodic memory encoding, whereas right PFC is more involved than left PFC in episodic memory retrieval. Recently it has been claimed that this description does not hold for nonverbal materials. Here we propose a more precise formulation of HERA than previously, and argue that there is sufficient evidence to conclude that HERA, as reformulated, is true for both verbal and non-verbal materials.
\end{abstract}

It has been known for some time now that structures within the two cerebral hemispheres do not contribute equally to mental activity [1]. The best known form of such hemispheric asymmetry is language: the left hemisphere is more involved in verbal processing than is the right. A more recent addition to the list of hemispheric asymmetries, although limited to the frontal regions of the brain, emerged from early PET studies of memory, and had to do with component processes of memory. Data from numerous studies showed that during learning of new material (encoding), left PFC tended to be more active than right $\mathrm{PFC}$, whereas during a subsequent recall or recognition test (retrieval), right PFC tended to be more active than left PFC. This general pattern of data, initially based only on a handful of studies [2,3], was dubbed the Hemispheric Encoding/Retrieval Asymmetry (HERA) model. Figure 1 illustrates the HERA asymmetry with data from a single study.

Although exceptions to HERA have been reported, numerous PET and fMRI studies have confirmed the existence of the general HERA pattern (for reviews see [4-7]). Recently, however, it has been suggested [8-14] that the asymmetry of PFC activations might reflect the nature of the materials involved in the comparison tasks (verbal versus non-verbal) rather than the memory processes (encoding versus retrieval). In light of these recent challenges, it is timely to re-evaluate the situation. We propose a more precise formulation of HERA, and argue that the challenges to it are based on confusion about what HERA is, and about what kinds of data are, and are not, relevant to it.

\section{HERA revised}

The confusion is partly attributable to the looseness of the early formulation of HERA, and partly to subtleties of the relations involved. Here, in keeping with similar suggestions $[10,15]$, we propose a stricter formulation of HERA. We suggest that the neuroanatomical data relevant to HERA should be based on a direct comparison of the two processes involved, with other variables, including materials, held constant.

Let us abbreviate encoding as Enc, retrieval as Ret, a given site in left PFC as L, and the corresponding site in right $\mathrm{PFC}$ as $\mathrm{R}$. And let us use the combination of tasks (Enc or Ret) and sites (L or R) to represent the level of (indirectly) measured neuronal activity at a given site in a given task.

Then the encoding component of HERA is supported if $(E n c \mathrm{~L}-\operatorname{Ret} \mathrm{L})>(E n c \mathrm{R}-\operatorname{Ret} \mathrm{R})$, and it is violated if $(E n c$ $\mathrm{L}-$ Ret $\mathrm{L})<($ Enc R-Ret R $)$. Similarly the retrieval component of HERA is supported if (Ret R-Enc R) > $(\operatorname{Ret} \mathrm{L}-E n c \mathrm{~L})$, and it is violated if $(\operatorname{Ret} \mathrm{R}-E n c \mathrm{R})<(\operatorname{Ret}$ $\mathrm{L}-E n c \mathrm{~L})$.

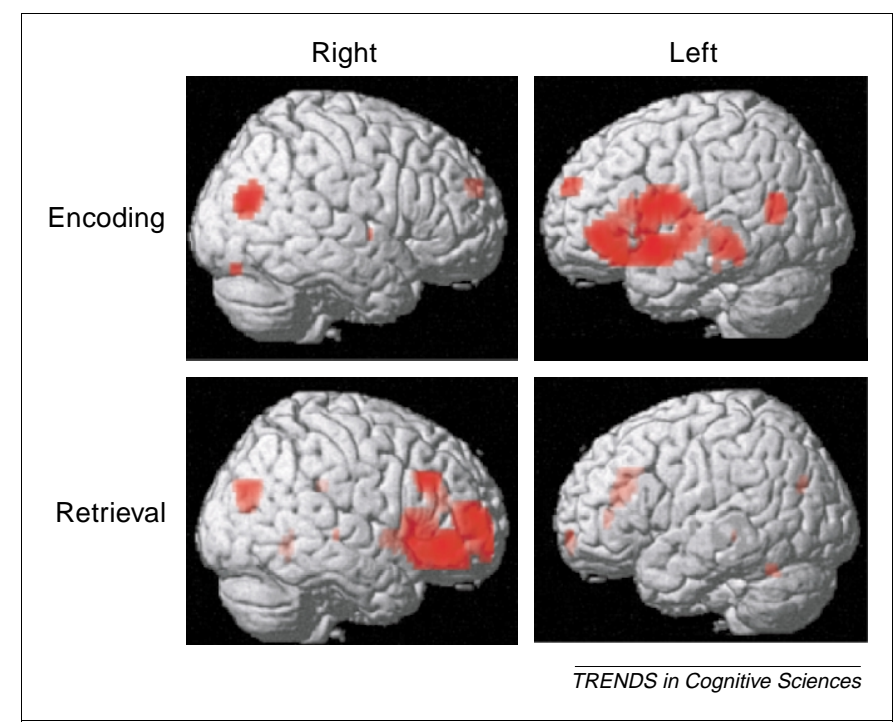

Fig. 1. Activations from a PET study of encoding and retrieval [40] are overlaid on a 3-D MRI brain. In this study, novelty and familiarity of the to-be-remembered items was held constant for both encoding and retrieval. Encoding refers to Encoding $>$ Retrieval, and Retrieval refers to Retrieval > Encoding (encoding and retrieval inequalities discussed in text). The pattern shows that encoding information into episodic memory activates the left hemisphere more than the right, whereas retrieval of information activates the right hemisphere more than the left. (Data re-analyzed and not previously published in this form.) 
Note five points about our proposal. First, to compute the difference in activity in each hemisphere, the proper reference condition for an encoding task is a retrieval task, and the proper reference condition for a retrieval task is an encoding task. Fixation, rest, and other non-active reference conditions are unsuitable because they fail to cleanly distinguish between encoding and retrieval: some incidental encoding or retrieval could occur during a rest or fixation condition (see also [16,17]). Second, absolute measures of brain activity during encoding and retrieval within each hemisphere, and their differences across hemispheres, are irrelevant to HERA. Rather, it is hemispheric asymmetries in the differences in activity between encoding and retrieval that are critical. Third, although comparisons between encoding and retrieval are required, our revised formulation of HERA does not imply that encoding and retrieval components need to be tested simultaneously; a study can focus on encoding, on retrieval, or both. Fourth, the proposed formulation is independent of the nature of the study or test materials, type of encoding or retrieval task, and other experimental variables. Fifth, there is nothing in the proposed formulation that rules out the possibility of the existence of other kinds of hemispheric asymmetry, including material-specific asymmetry, or any other kind of regularity in the data.

\section{Process and material asymmetries}

As material-specific hemispheric asymmetries are commonplace, it is not surprising that comparable findings have also been obtained in memory-related tasks. Kelley et al. [8] observed that, in an encoding task, activity was greater in left than right PFC when subjects studied words, greater in right than left PFC when subjects studied non-famous faces, and equivalent in left and right PFC when they studied nameable objects. Wagner et al. [9] compared verbal and non-verbal materials, while holding constant the task (either encoding or retrieval) and observed prominent material-specific lateralization of activation: left for verbal and right for non-verbal.

These and other similar findings [10,11,18-21] have led to criticisms of HERA and to rival proposals. One such is that the main determinant of the hemispheric involvement in memory tasks is the type of to-be-remembered materials, and processes such as encoding and retrieval serve at best only to modulate such determination $[1,8,9,13,14,18]$. Another is that the hemispheric asymmetry between encoding and retrieval is apparent only: it might reflect nothing more than the asymmetry between the more verbally oriented processing during encoding and the less verbally oriented processing during retrieval $[11,12,22]$.

The common theme here seems to be that there is only one kind of hemispheric asymmetry possible, or valid, or allowed. Therefore, if it is not encoding versus retrieval, then it must be something else instead. We find the excessive parsimony inherent in this kind of thinking unwarranted. There is no reason why process-specific and material-specific hemispheric asymmetries, as well as other types, could not co-exist (as demonstrated below) in $\mathrm{PFC}$, or more generally in the entire brain.

Given two kinds of asymmetry, one defined by processes and the other by materials, it is sensible of course to explore their relation. This can be done experimentally in a situation in which both are systematically manipulated. A study by Nyberg et al. [23] illustrates this approach. In this study, subjects were presented with a series of sentences or photographs for encoding and subsequent recognition. The data were analyzed using the multivariate partial least squares (PLS) method [24]. PLS is similar to other multivariate techniques such as factor and principal components analysis, and identifies orthogonal latent variables which represent the largest sources of experimental variance. In the study by Nyberg et al. [23], the first latent variable distinguished processing of verbal stimuli (sentences) from processing of non-verbal stimuli (photographs). The second latent variable, whose variance was orthogonal to that of the first, identified a pattern of brain activation that was related to encoding and retrieval of both verbal and non-verbal information and was consistent with the HERA model.

Thus, the Nyberg et al. study [23] demonstrates that material-specificity can occur independently of processspecificity, and that once material-specific hemispheric asymmetries are accounted for, a general encoding and retrieval pattern of brain activation that is consistent with the HERA model does exist. A concrete example of the independence of the two kinds of asymmetry is given in (Box 1).

\section{Is HERA valid?}

HERA is supported by large amounts of data. Data are consistent in showing that right $\mathrm{PFC}$ is biased for retrieval of verbal materials [4-7] as well as nonverbal materials $[10,23,25,26]$. It has been suggested that this bias is largely, or perhaps even entirely, attributable to the general mental set towards temporally guided retrieval, or retrieval mode [6]. If true, this hypothesis would explain why retrieval is frequently left-lateralized [27-29] under conditions where retrieval mode is held constant.

Data also show that left PFC is biased for encoding of verbal materials [2,3] as well as non-verbal materials [12,23,26,30-35]. The apparently critical findings of rightlateralization of encoding of non-verbal materials $[8,9,18]$ have no relevance to HERA. They provide good evidence in support of material-specific hemispheric asymmetry, but, because encoding and retrieval processes were not systematically varied and their interaction with hemispheres was not examined in these studies, the data are neutral with respect to HERA. We, therefore, conclude that the HERA model holds for both verbal and non-verbal materials.

HERA has several weaknesses, including the fact that exceptions to it occur every now and then in the form of data from specific studies. Some of these were noted already in the earliest days of the model [2,3]. But as an economical description of large amounts of data from different laboratories, HERA has retained its heuristic 
Box 1. Process-specific versus material-specific asymmetry

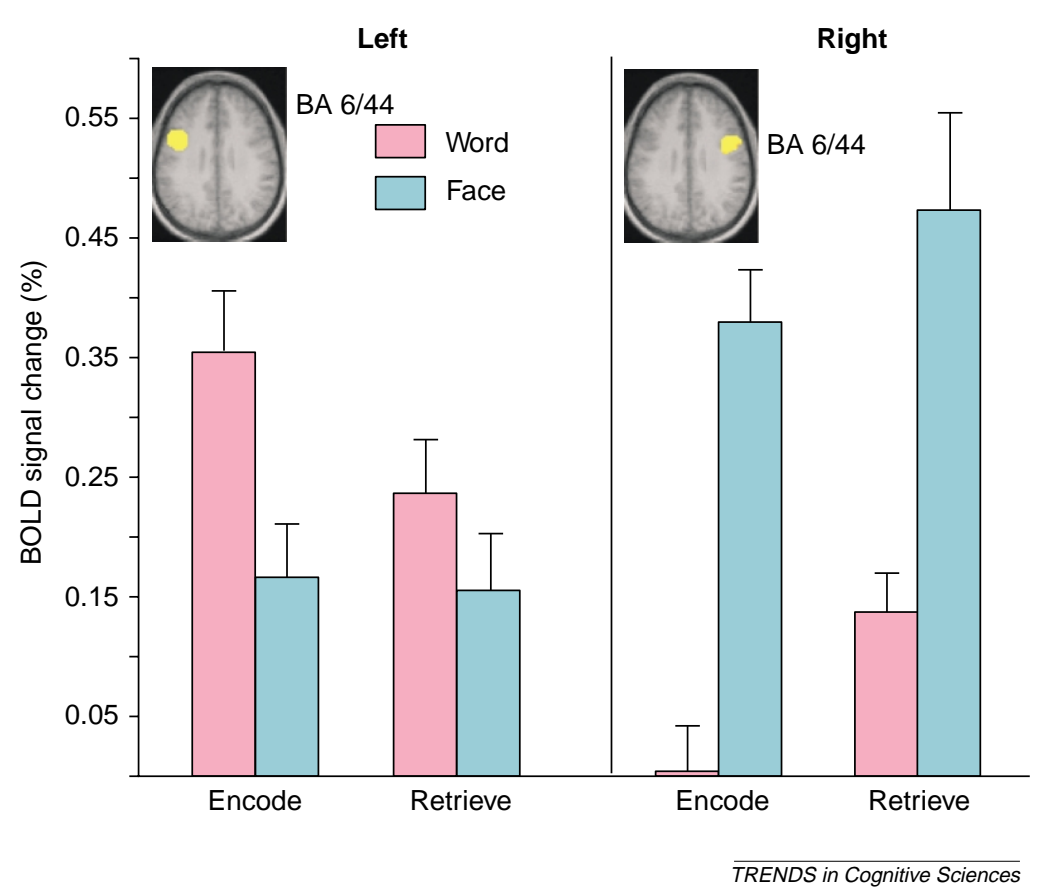

Fig. I. Percentage fMRI BOLD signal change in the left and right inferior frontal gyrus (BA 6/44) as a function of type of material (words vs. faces) and type of processing (encoding vs. retrieval). (Redrawn with permission from Ref. [10].)

The demonstration that encoding verbal material tends to be leftlateralized whereas encoding of non-verbal material tends to be rightlateralized has led some researchers to conclude that a neuropsychologically based material-specific asymmetry model should be preferred over HERA $[8,9,11-14]$. A study by McDermott et al. [10] demonstrates the logic of this thinking. In this fMRI study, subjects intentionally encoded words and faces and were later tested with a yes/no recognition test. The authors observed that a left PFC site was more active for verbal than non-verbal encoding and retrieval, whereas a right PFC site was more active for non-verbal than verbal encoding and retrieval (see Fig. I). The data from this figure are reproduced in Table I.

An examination of the marginal means of the signal changes supports the conclusion of material-specificity. In the left PFC region, verbal encoding and retrieval activity $(0.30)$ is greater than non-verbal encoding and retrieval activity (0.15), whereas in the right PFC region, non-verbal encoding and retrieval activity $(0.42)$ is greater than verbal encoding and retrieval activity (0.07).

However, if the same data are tested with the HERA inequalities, a different pattern emerges. Encoding activity, in relation to retrieval activity, is higher in left PFC than right PFC for both verbal and nonverbal materials: $0.10>-0.14$, and $0.02>-0.08$, respectively. Retrieval

usefulness. Such usefulness extends to experimental paradigms and approaches beyond neuroimaging $[13,14,36,37]$. Especially intriguing are the results reported by Rossi and colleagues [37] (see Box 2).

\section{The future of HERA}

Outstanding questions for future research have to do with the basis for the asymmetry. Why should the asymmetry exist? Gazzaniga [1] has observed that the left hemisphere differs from the right in mnemonic functions, with the left more specialized for semantic processing and the right for episodic memory. It is activity, in relation to encoding activity, is higher in right PFC than left PFC for both verbal and non-verbal materials, $0.14>-0.10$, and $0.08>$ -0.02 , respectively.

These results demonstrate that both a material-specific and a process-specific asymmetry can even appear in the same region. The two kinds of asymmetry certainly can appear in different regions. It follows, therefore, that material-specific and process-specific models of hemispheric asymmetry can co-exist. There is no need to argue for the existence or reality of only one, or for the primacy of one over the other.

Table I. Percentage signal change in left and right PFC during verbal and non-verbal encoding and retrieval.

\begin{tabular}{|llll|}
\hline & & Words & Faces \\
\hline Left PFC & Encoding & 0.35 & 0.16 \\
& Retrieval & 0.25 & 0.14 \\
Right PFC & Mean & 0.30 & 0.15 \\
& Encoding & 0.00 & 0.38 \\
& Retrieval & 0.14 & 0.46 \\
& Mean & 0.07 & 0.42 \\
\hline
\end{tabular}

known that episodic encoding relies heavily on semantic processes, so it is reasonable to imagine that leftlateralization of encoding is attributable to semantic processing of the incoming and on-line information. The right lateralization of episodic retrieval has been accounted for in terms of 'retrieval mode' [6]. Retrieval mode refers to a neurocognitive set, a necessary condition that sets the stage for episodic remembering. But why is the left hemisphere specialized for semantic memory (and episodic encoding) and the right for episodic retrieval? We might speculate that, earlier in evolution, mental functions were fewer than today and 


\section{Box 2. rTMS and HERA}

Although neuropsychological patients with frontal damage are not amnesic, they do, as a rule, suffer from memory impairment [38]. A natural question to ask, therefore, is whether brain-damaged patients with left prefrontal lesions are more impaired during encoding than retrieval, and whether brain-damaged patients with right prefrontal lesions are more impaired during retrieval than encoding. Because neuropsychological patient data cannot be used to distinguish between encoding and retrieval processes, the question has no answer.

Recently, however, a new technique called repetitive Transcranial Magnetic Stimulation ( $r$ TMS) is providing the ability to safely disrupt the activity of complex cortical neural networks with rapid discharges of trains of magnetic impulses [39]. This technique, in effect, can be used to produce 'reversible cortical lesions' in healthy individuals, allowing for the evaluation of the role of a particular brain region in mental activity.

Rossi et al. [37] have applied rTMS to the examination of HERA. In their study, subjects incidentally encoded and were later tested on recognition of a series of complex colored magazine photographs while receiving rTMS. Their results supported the HERA model by demonstrating that during encoding, rTMS over the left PFC hindered memory performance to a greater extent than rTMS over the right PFC, whereas during retrieval, rTMS over the right PFC hindered memory performance to a greater extent than rTMS over the left PFC (see Fig. I).

These results directly implicate left and right PFC in encoding and retrieval processes, respectively, exactly as described by HERA. They

their cortical basis was bilateral. As more sophisticated mental capacities evolved there was a growing demand for cortical space [1]. The solution to this problem was hemispheric specialization: new functions were taken over by one hemisphere, at the cost of displacing earlier functions which, however, were retained in the other hemisphere.

\section{Acknowledgements}

R.H. is supported by a McDonnell Foundation investigator initiated grant to A.R. McIntosh and a Tanenbaum postdoctoral fellowship. L.N. is supported by the Swedish Research Council. E.T. is supported by a foundation of Anne and Max Tanenbaum in support of research in cognitive neuroscience and by the Natural Sciences and Engineering Research Council of Canada.

\section{References}

1 Gazzaniga, M.S. (2000) Cerebral specialization and interhemispheric communication: does the corpus callosum enable the human condition? Brain 123, 1293-1326

2 Tulving, E. et al. (1994) Hemispheric encoding/retrieval asymmetry in episodic memory: positron emission tomography findings. Proc. Natl. Acad. Sci. U. S. A. 91, 2016-2020

3 Nyberg, L. et al. (1996) PET studies of encoding and retrieval: the HERA model. Psychonomic Bull. Rev. 3, 135-148

4 Desgranges, B. et al. (1998) The functional neuroanatomy of episodic memory: the role of the frontal lobes, the hippocampal formation, and other areas. NeuroImage 8, 198-213

5 Cabeza, R. and Nyberg, L. (2000) Imaging cognition II: an empirical review of 275 PET and fMRI studies. J. Cogn. Neurosci. 12, 1-47

6 Lepage, M. et al. (2000) Prefrontal cortex and episodic memory retrieval mode. Proc. Natl. Acad. Sci. U. S. A. 97, 506-511

7 Fletcher, P.C. and Henson, R.N. (2001) Frontal lobes and human memory: insights from functional neuroimaging. Brain 124, 849-881

8 Kelley, W.M. et al. (1998) Hemispheric specialization in human dorsal frontal cortex and medial temporal lobe for verbal and nonverbal memory encoding. Neuron 20, 927-936

9 Wagner, A.D. et al. (1998) Material-specific lateralization of prefrontal activation during episodic encoding and retrieval. NeuroReport 9, 3711-3717

10 McDermott, K.B. et al. (1999) Set- and code-specific activation in

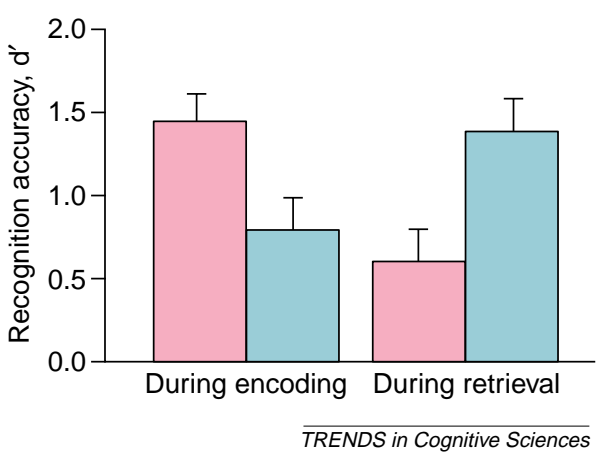

Fig. I. Recognition accuracy, d', following 'reversible lesions' produced by rTMS of right (violet) and left (green) dorsolateral PFC during encoding and retrieval of complex pictures. The results reveal a hemispheric asymmetry in memory performance. (Redrawn with permission from Ref. [37].)

thus provide converging support for the HERA model. Especially interesting is the fact that the support came from a study using nonverbal materials. frontal cortex: an fMRI study of encoding and retrieval of faces and words. J. Cogn. Neurosci. 11, 631-640

11 Lee, A.C. et al. (2000) Asymmetric frontal activation during episodic memory: the effects of stimulus type on encoding and retrieval. Neuropsychologia 38, 677-692

12 Owen, A.M. et al. (1996) Memory for object features versus memory for object location: a positron-emission tomography study of encoding and retrieval processes. Proc. Natl. Acad. Sci. U. S. A. 93, 9212-9217

13 Miller, M.B. et al. (2002) Hemispheric encoding asymmetry is more apparent than real. J. Cogn. Neurosci. 14, 702-708

14 Epstein, C.M. et al. (2002) Asymmetries of prefrontal cortex in human episodic memory: effects of transcranial magnetic stimulation on learning abstract patterns. Neurosci. Lett. 320, 5-8

15 Duncan, J. and Owen, A.M. (2000) Common regions of the human frontal lobe recruited by diverse cognitive demands. Trends Neurosci. $23,475-483$

16 Sergent, J. et al. (1992) Positron emission tomography study of letter and object processing: empirical findings and methodological considerations. Cereb. Cortex 2, 68-80

17 Stark, C.E.L. and Squire, L.R. (2001) When zero is not zero: the problem of ambiguous baseline conditions in fMRI. Proc. Natl. Acad. Sci. U. S. A. 98, 12760-12766

18 Golby, A.J. et al. (2001) Material-specific lateralization in the medial temporal lobe and prefrontal cortex during memory encoding. Brain $124,1841-1854$

19 Kirchhoff, B.A. et al. (2000) Prefrontal-temporal circuitry for episodic encoding and subsequent memory. J. Neurosci. 20, 6173-6180

20 Brewer, J.B. et al. (1998) Making memories: brain activity that predicts how well visual experience will be remembered. Science 281, 1185-1187

21 Wagner, A.D. et al. (1998) Building memories: remembering and forgetting of verbal experiences as predicted by brain activity. Science 281, 1188-1191

22 Lee, A.C.H. et al. (2000) Episodic memory meets working memory in the frontal lobe: functional-neuroimaging studies of encoding and retrieval. Crit. Rev. Neurobiol. 14, 165-198

23 Nyberg, L. et al. (2000) Large scale neurocognitive networks underlying episodic memory. J. Cogn. Neurosci. 12, 163-173

24 McIntosh, A.R. et al. (1996) Spatial pattern analysis of functional brain images using partial least squares. NeuroImage 3, 143-157

25 Grady, C.L. et al. (2001) An examination of the effects of stimulus type, encoding task, and functional connectivity on the role of right prefrontal cortex in recognition memory. NeuroImage 14, 556-571 
26 Johnson, M.K. et al. (2003) FMRI evidence for an organization of prefrontal cortex by both type of process and type of information. Cereb. Cortex 13, 265-273

27 Henson, R.N. et al. (2000) Confidence in recognition memory for words: dissociating right prefrontal roles in episodic retrieval. J. Cogn. Neurosci. 12, 913-923

28 Henson, R.N.A. et al. (1999) Recollection and familiarity in recognition memory: an event-related functional magnetic resonance imaging study. J. Neurosci. 19, 3962-3972

29 Rugg, M.D. et al. (2003) Neural correlates of retrieval processing in the prefrontal cortex during recognition and exclusion tasks. Neuropsychologia 41, 40-52

30 Bernstein, L.J. et al. (2002) The effect of encoding strategy on the neural correlates of memory for faces. Neuropsychologia 40, 86-98

31 Downes, J.J. et al. (2001) Prefrontal cortical activation during wordassociative, face- associative, and word-face-associative encoding. Brain Cogn. 47, 69-72

32 Iidaka, T. et al. (2000) Functional asymmetry of human prefrontal cortex in verbal and non-verbal episodic memory as revealed by fMRI. Cogn. Brain Res. 9, 73-83
33 Buckner, R.L. et al. (1998) Functional-anatomic correlates of object priming in humans revealed by rapid presentation event-related fMRI. Neuron 20, 285-296

34 Haxby, J.V. et al. (1996) Face encoding and recognition in the human brain. Proc. Natl. Acad. Sci. U. S. A. 93, 922-927

35 Wagner, A.D. et al. (1997) Semantic repetition priming for verbal and pictorial knowledge: a functional MRI study of left inferior prefrontal cortex. J. Cogn. Neurosci. 9, 714-726

36 Blanchet, S. et al. (2001) New questions on the hemispheric encoding/ retrieval asymmetry (HERA) model assessed by divided visual-field tachistoscopy in normal subjects. Neuropsychologia 39, 502-509

37 Rossi, S. et al. (2001) Prefrontal cortex in long-term memory: an "interference" approach using magnetic stimulation. Nat. Neurosci. 4, 948-952

38 Wheeler, M.A. et al. (1995) Frontal lobe damage produces memory impairment. J. Int. Neuropsychol. Soc. 1, 525-536

39 Hallett, M. (2000) Transcranial magnetic stimulation and the human brain. Nature 406, 147-150

40 Düzel, E. et al. (1999) Task-related and item-related brain processes of memory retrieval. Proc. Natl. Acad. Sci. U. S. A. 96, 1794-1799

\section{Could you name the most significant papers published in life sciences this month?}

Updated daily, Research Update presents short, easy-to-read commentary on the latest hot papers, enabling you to keep abreast with advances across the life sciences.

Written by laboratory scientists with a keen understanding of their field, Research Update will clarify the significance and future impact of this research.

Our experienced in-house team are under the guidance of a panel of experts from across the life sciences who offer suggestions and advice to ensure that we have high calibre authors and have spotted the 'hot' papers.

Visit the Research Update daily at http://update.bmn.com and sign up for email alerts to make sure you don't miss a thing.

This is your chance to have your opinion read by the life science community, if you would like to contribute contact us at research.update@elsevier.com 